\title{
Anisotropic Heisenberg model on hierarchical lattices with aperiodic interactions: a renormalization-group approach
}

\author{
N. S. Branco, 1, 囷 J. Ricardo de Sousa, ${ }^{2,3}$ and Angsula Ghosh ${ }^{2}$ \\ ${ }^{1}$ Departamento de Física, Universidade Federal de Santa Catarina, 88040-900, Florianópolis, SC, Brazil \\ ${ }^{2}$ Departamento de Física, UFAM, 3000-Japiim, 69077-000 Manaus, AM, Brazil \\ ${ }^{3}$ Departamento de Física, ICEx, Universidade Federal de Minas Gerais, 30123-970, Belo Horizonte-MG, Brazil
}

(Dated: November 9, 2018)

\begin{abstract}
Using a real-space renormalization-group approximation, we study the anisotropic quantum Heisenberg model on hierarchical lattices, with interactions following aperiodic sequences. Three different sequences are considered, with relevant and irrelevant fluctuations, according to the LuckHarris criterion. The phase diagram is discussed as a function of the anisotropy parameter $\Delta$ (such that $\Delta=0$ and $\Delta=1$ correspond to the isotropic Heisenberg and Ising models, respectively). We find three different types of phase diagrams, with general characteristics: the isotropic Heisenberg plane is always an invariant one (as expected by symmetry arguments) and the critical behavior of the anisotropic Heisenberg model is governed by fixed points on the Ising-model plane. Our results for the isotropic Heisenberg model show that the relevance or irrelevance of aperiodic models, when compared to their uniform counterpart, is as predicted by the Harris-Luck criterion. A low-temperature renormalization-group procedure was applied to the classical isotropic Heisenberg model in two-dimensional hierarchical lattices: the relevance criterion is obtained, again in accordance with the Harris-Luck criterion.
\end{abstract}

PACS numbers:

\section{INTRODUCTION}

The investigation of systems displaying inhomogeneous or disordered interactions is an active field of research [? ? ]. From the experimental point of view, many of the materials found in nature come with impurities; also, modern techniques are able to build materials with controlled composition, such that two or more different atoms are combined in a given order. Theoretically, one may be concerned with possible changes on the critical behavior of systems, with the introduction of random disorder or inhomogeneuos deterministic interactions, when compared to their homogeneous counterpart [? ]. For quenched random disorder, the Harris criterion [? ] states that, if the pure-system's specific-heat exponent, $\alpha$, is positive (negative), the critical behavior of the disordered model is diffferent from (the same as for) the pure model.

The discovery of quasi-crystals [? ] has motivated an intense research on the behavior of models with interactions following aperiodic sequences [? ]: numerical [? ] as well as analytical results [? ? ] have been obtained. Many works concentrated on classical models, like Ising and Potts [? ? ] ones, but some attention has been drawn to quantum models in one dimension [? ? ? ? ? ? ]. In Ref. ? the ground-state properties of a two-dimensional quantum model have been analyzed. However, no work has focused on the finite-temperature critical behavior of quantum models in dimensions two or above, to the best of our knowledge.

*Electronic address: nsbranco@fisica.ufsc.br
A convenient model to address the role played by quantum effects and aperiodicity on critical phenomena is the anisotropic Heisenberg one, with interactions following aperiodic and deterministic sequences. On the other hand, the way these sequences are constructed and the idea behind renormalization-group calculations make hierarchical lattices a natural choice for the study. Therefore, in this work we treat the anisotropic ferromagnetic Heisenberg model on four different hierarchical lattices, with different Hausdorff fractal dimenions. Three different aperiodic sequences are treated, corresponding to bounded and unbounded fluctuations. We will study here mainly the relevance of the introduction of aperiodicity, in the renormalization-group sense. This aspect is generally addressed by the so-called Harris-Luck criterion [? ]. According to this criterion, the relevance of a given aperiodic sequence is connected to the crossover exponent, $\phi$, given by [? ? ]:

$$
\phi=1-d_{a} \nu_{0}(1-\omega),
$$

where $d_{a}$ is the dimension the aperiodic sequence acts on, $\nu_{0}$ is the correlation lenght's critical exponent of the pure model and $\omega$ is the fluctuation exponent. This exponent is defined through $g \sim N^{\omega}$, where $g$ is the fluctuation in the number of a given letter of the sequence (below, we discuss this point further). For $\phi>0$, the critical behavior of the aperiodic and uniform models are in different universality classes. For $\phi<0$, both aperiodic and uniform models have the same set of critical exponents. For the marginal case, $\phi=0$, critical exponents depend on the ratio between the two interaction constants [? ? ]; we will not duscuss this case further. Our choices of hierarchical lattices and aperiodic sequences allow for values of $\phi$ greater os smaller than zero, as well as for differ- 
ent fractal dimensions of the lattice. The aperiodicity is chosen such that $d_{a}=1$ in all cases we study.

This work is organized as follows. In the next section we review some basic concepts of aperiodic sequences, which will be important to our work. In Section III) we define the model and outline the real-space renormalizationgroup approach we use and in Section IV we present our results. In Section $\nabla$ we discuss and summarize the results.

\section{APERIODIC SEQUENCES}

The aperiodic sequences used in this work are obtained by the iteration of substitutuon rules working on an alphabet. Each letter of the alphabet is replaced by a sequence of letters and words are formed at each stage. We will be concerned with a two-letter alphabet, $A$ and $B$, and the usual convention is that the initial word is $A$. More specifically, we will study the following aperiodic sequences:

(i) $A \rightarrow A B ; \quad B \rightarrow A A$, i.e., from a given word of the sequence, the next word is obtained by substituting $A B$ for every letter $A$ and $A A$ for every letter $B$ in the previous word. The first stagess of this sequence are $A \rightarrow A B \rightarrow A B A A \rightarrow A B A A A B A B \rightarrow \ldots$ This is the so-called period-doubling sequence.

(ii) $A \rightarrow A B B ; \quad B \rightarrow A A A$. The first stages of this sequence are: $A \rightarrow A B B \rightarrow A B B A A A A A A \rightarrow$ $\ldots$;

(iii) $A \rightarrow A A B ; \quad B \rightarrow A A A$. In this case, the first words of the sequence are: $A \rightarrow A A B \rightarrow$ $A A B A A B A A A \rightarrow \ldots$.

The geometrical characteristics of these sequences are obtained from the substitution matrix $\mathcal{M}$, which connects the number of letters $A$ and $B$ after one application of the iteration rule, namely:

$$
\left(\begin{array}{l}
N_{A}^{(n+1)} \\
N_{B}^{(n+1)}
\end{array}\right)=\mathcal{M}\left(\begin{array}{c}
N_{A}^{(n)} \\
N_{B}^{(n)}
\end{array}\right)
$$

Therefore, after $n$ iterations the total number of letters in the word, $N^{(n)}$, is given by: $N^{(n)} \equiv N_{A}^{(n)}+N_{B}^{(n)}=$ $\mathcal{M}^{n} N^{(0)}$, where $N_{A}^{(n)}$ and $N_{B}^{(n)}$ are the number of letters $A$ and $B$ after $n$ iterations, respectively, and $N_{A}^{(0)}=1$ and $N_{B}^{(0)}=0$ for the initial word.

The substitution matrices for the three aperiodic sequences defined above are:

$$
\begin{aligned}
& \text { (i) } \mathcal{M}=\left(\begin{array}{ll}
1 & 2 \\
1 & 0
\end{array}\right) ; \\
& \text { (ii) } \mathcal{M}=\left(\begin{array}{ll}
1 & 3 \\
2 & 0
\end{array}\right) ; \\
& \text { (iii) } \mathcal{M}=\left(\begin{array}{ll}
2 & 3 \\
1 & 0
\end{array}\right) \text {. }
\end{aligned}
$$

The total number of letters grows exponentially with the number of iterations $n$ :

$$
N \sim \lambda_{1}^{n}, n \rightarrow \infty
$$

where $N \equiv \lim _{n \rightarrow \infty} N^{(n)}$ and $\lambda_{1}$ is the greater eigenvalue of $\mathcal{M}$. For the three sequences studied in this work, this exponential growth is valid for all $n$ and $\lambda_{1}=2$ for sequence $(i)$ and $\lambda_{1}=3$ for sequences (ii) and (iii). One can define the fluctuation in a given letter, say $A$, as $g^{(n)}=N_{A}^{(n)}-p_{A} N^{(n)}$, where $p_{A}$ is the fraction of letters $A$ in the infinite word, i.e, after $n$ applications of the iteration rules, with $n \rightarrow \infty$. The fractions $p_{A}$ and $p_{B}$ are proportional to the first and second entries, respectively, of the eigenvector corresponding to the greater eigenvalue. It is possible to show that:

$$
g \sim \lambda_{2}^{n}, n \rightarrow \infty,
$$

where $g \equiv \lim _{n \rightarrow \infty} g^{(n)}$ and $\lambda_{2}$ is the smaller eigenvalue of $\mathcal{M}$. Therefore, using Eqs. (3) and (4), one can show that:

$$
g \sim N^{\omega}, \omega=\frac{\ln \left|\lambda_{2}\right|}{\ln \lambda_{1}} .
$$

The exponent $\omega$ is crucial for the crossover exponent, as outlined in the previous section. Its value is $w=0$, $\ln (2) / \ln (3)$ and 0 for sequences $(i),($ ii $)$ and (iii), respectively, as can be easily calculated from their substitution matrices. We will disccus the results for the crossover exponent in Section IV]

\section{MODEL AND FORMALISM}

The reduced Hamiltonian of the anisotropic Heisenberg model is given by:

$$
-\beta \mathcal{H}=\sum_{<i, j>} K_{i j}\left[(1-\Delta)\left(\sigma_{i}^{x} \sigma_{j}^{x}+\sigma_{i}^{y} \sigma_{j}^{y}\right)+\sigma_{i}^{z} \sigma_{j}^{z}\right],
$$

where $\beta=1 / k_{B} T, k_{B}$ being the Boltzmann constant and $T$ the temperature, $\sigma_{i}^{\alpha}$ is the component $\alpha$ of a spin- $1 / 2$ Pauli matrix on site $i, 0 \leq \Delta \leq 1$ ( $\Delta=0$ corresponds to the isotropic Heisenberg model and $\Delta=1$ to the Ising model), the sum is over all first-neighbor bonds on a hierarchical lattice and the exchange constants $K_{i j}=\beta J_{i j}$ follow an aperiodic sequence in a given direction of the lattice. See Figs. 1 and 2 for examples of hierarchical lattices with $b=2$ and $b=3$, respectively: these lattices are built of $b^{d-1}$ bonds connected in parallel, each one consisting of $b$ bonds connected in series, where $d$ is the fractal dimension of the lattice. In this work, we have treated lattices with $d=2$ and $d=3$.

We use a real-space renormalization-group approach; a partial trace is taken over internal spins on suitable finite lattices and a renormalized Hamiltonian is obtained, namely:

$$
\begin{aligned}
e^{-(\beta \mathcal{H})^{\prime}} & =\exp \left\{K_{12}^{\prime}\left[\left(1-\Delta^{\prime}\right)\left(\sigma_{1}^{x} \sigma_{2}^{x}+\sigma_{1}^{y} \sigma_{2}^{y}\right)+\sigma_{1}^{z} \sigma_{2}^{z}\right]\right\} \\
& \equiv \operatorname{Tr}_{\{\sigma\}} e^{-\beta \mathcal{H}}
\end{aligned}
$$




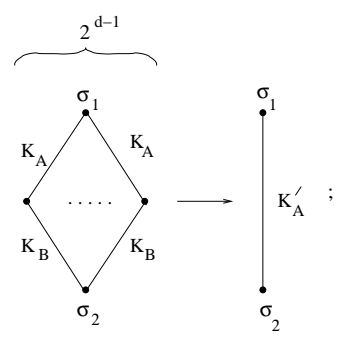

(a)



(b)
FIG. 1: Hierarchical lattice with $b=2$, suitable for the study of the period-doubling sequence (sequence $(i)$; see text). We show the renormalization for the coupling constant $K_{A}$ (part $(a))$ and for the coupling constant $K_{B}$ (part (b)). Note that the construction of the hierarchical lattice is made in the reverse order of the renormalization-group procedure.

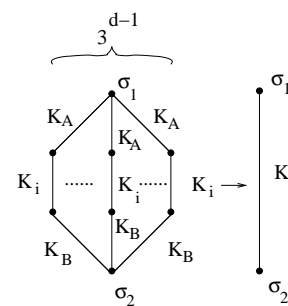

(a)

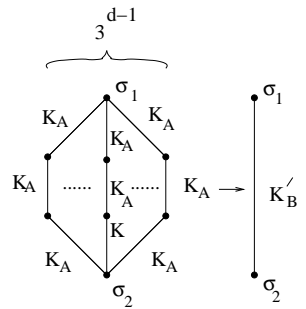

(b)
FIG. 2: Hierarchical lattice with $b=3$, suitable for the study of sequences (ii) and (iii) (see text). We show the renormalization for the coupling constant $K_{A}$ (part $(a)$ ), where $K_{i}=K_{B}$ for sequence $(i i)$ and $K_{i}=K_{A}$ for sequence $(i i i)$, and for the coupling constant $K_{B}$ (part $\left.(b)\right)$.

where $K_{12}^{\prime}=K_{A}^{\prime}$ in Figs. 1 $(a)$ and 2 $(a)$ and $K_{12}^{\prime}=K_{B}^{\prime}$ in Figs. $1(b)$ and $2(b), \operatorname{Tr}_{\{\sigma\}}$ is a partial trace, taken over all spins in Figs. 11 and 2, except $\sigma_{1}$ and $\sigma_{2}$, and $\beta \mathcal{H}$ is the reduced Hamiltonian of the cell on the left-hand sides of Fig. 1 ( $a$ ) and 1 (b) and Fig. 2 (a) and 2 (b). The method to calculate the partial trace in Eq. (7) for quantum systems was introduced in Ref. ? and an important simplification was proposed in Ref. ? , where the whole process is explained in detail. This method has been successfully applied in the study of ferromagnetic, antiferromagnetic and spin-glass quantum models. The formalism is specially suitable to obtain multidimensional phase diagrams and qualitative results, indicating universality classes and possible crossover phenomena. It is worth mentioning that, although $\Delta$ is initially uniform and the aperiodicity acts only on the interaction parameter $K$, after the first iteration of the renormalization-group the anisotropy is no longer the same for every bond. This fact has to be taken into account when deriving the recursion relations. We refer the reader to Refs. ? and ? for details.

\section{RESULTS AND DISCUSSION}

Some features are common to all three sequences: the isotropic Heisenberg model $(\Delta=0)$ is an invariant subspace, in the renormalization-group sense. The same applies for the Ising model $(\Delta=1)$. The reason is that the introduction of exchange constants with different values does not change the symmetry of these two models. Therefore, the flow will not leave the corresponding subspaces. Moreover, the critical behavior for $0<\Delta<1$ will be determined by the stability of the Ising-model non-trivial fixed points, i.e, the flow for any initial value of $\Delta$ in that range is towards the $\Delta=1$ subspace. The Ising model with aperiodic interactions has already been treated and our results for $\Delta=1$ agree with those in Ref. ? . Particularly, the stability of the uniform fixed points, with respect to the introduction of aperiodicity, is in accordance with the Harri-Luck criterion. Therefore, from now on we will restrict ourselves to the isotropic Heisenberg model subspace.

In the renormalization-group framework, the stability of fixed points is given by the eigenvalues of the matrix of the linearized renormalization-group equations (LRGE) [? ]. Since the relevant fixed points (filled squares in Fig. 3) are the non-trivial ones, one of these eigenvalues is always greater than one and corresponds to the flux along the uniform sub-space (traced lines in Fig. 31). In this figure we show the qualitative picture we obtain for irrelevant (part $(a)$ ) and relevant (part $(b)$ ) aperiodic sequences for $d=3$. The uniform model corresponds to the straight line at $45^{\circ}$, where $K_{A}=K_{B}$; the fixed point is always unstable along this line. The relevance of the aperiodicity is given by the stability along the other direction (continuous lines leaving the fixed points in Fig. 3): in part $(a)$ of the figure, the aperiodicity does not change the critical behavior, compared to the uniform model, while in part $(b)$ a new universality class emerges when aperiodicity is introduced. Note that the phase diagrams for lattices with $d=2$ are qualitative different from the ones in Fig. 3, the non-trivial fixed points are at zero temperature $(K=\infty)$ and, therefore, the "aperiodic" direction is not physically accessible in these cases. Nevertheless, the relevance of the aperiodicity is correctly described by the renormalization-group formalism, as we will see below.

A technical point is worth mentioning here. For all sequences we treat in this work the structure of the matrix of the LRGE, evaluated at the uniform fixed point, $K^{*} \equiv K_{A}^{*}=K_{B}^{*}$, is:

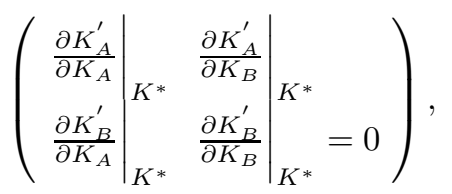

Since:

$$
\partial K_{B}^{\prime} /\left.\partial K_{A}\right|_{K^{*}}=\partial K_{A}^{\prime} /\left.\partial K_{A}\right|_{K^{*}}+\partial K_{A}^{\prime} /\left.\partial K_{B}\right|_{K^{*}},
$$


the eigenvalues of the above matrix are:

$$
\Lambda_{1}=\left.\frac{\partial K_{B}^{\prime}}{\partial K_{A}}\right|_{K^{*}} ; \Lambda_{2}=-\left.\frac{\partial K_{A}^{\prime}}{\partial K_{B}}\right|_{K^{*}}
$$

The former corresponds to the uniform model, as discussed previously, and, therefore, is always greater than 1. The absolute value of the latter eigenvalue determines the relevance of the aperiodicity.

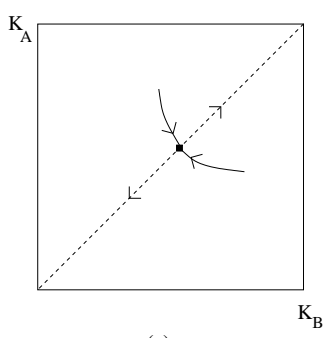

(a)

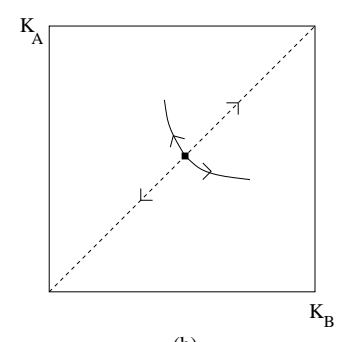

(b)
FIG. 3: Qualitative phase diagram for hierarchical lattices with $d=3$. (a) Renormalization-group flux for irrelevant aperiodic sequences; (b) Renormalization-group flux for relevant aperiodic sequences. The straight lines at 45 degrees represent the uniform model, $K_{A}=K_{B}$, and the fixed point is relevant along this direction.

For classical models, like the Ising and Potts ones [? ], the matrix of the LRGE is proportional to the transpose of the substitution matrix (see Eq. (2) above), $\mathcal{M}^{\mathrm{T}}$. This property still holds true for quantum models and for aperiodic sequences such that the hierarchical lattices which renormalizes into $K_{A}^{\prime}$ is symmetric with respect to the exchange of the iterations $K_{A}$ and $K_{B}$, as in part ( $a$ ) of Fig. 1. This brings an important simplification for the calculation of the former matrix, since the proportionality factor can be obtained from the recursion relation for the uniform model, i.e, from the renormalization of $K_{B}$. Then, one can obtain the other two elements, $\partial K_{A}^{\prime} /\left.\partial K_{A}\right|_{K^{*}}$ and $\partial K_{A}^{\prime} /\left.\partial K_{B}\right|_{K^{*}}$, from the substitution matrix, with no need to work out the recursion relation for the aperiodic model (this relation involves bonds with different values, which may make it cumbersome to be calculated). Note that, while the proportinality between the matrix of the LRGE and $\mathcal{M}^{\mathrm{T}}$ is always true for classical models, it fails for quantum models when the lattice is not symmetric with respect to the exchange of the iterations $K_{A}$ and $K_{B}$ (like the one in part (a) of Fig. 22 for example).

For the hierarchical lattices with $b=3$, we have to resort to the Migdal-Kadanoff approximation, since the number of sites involved in the renormalization is rather large. This approximation is equivalent to treating the cell "by pieces", i.e., to renormalizating first the $b$ bond in series and then combining the $b^{d-1}$ renormalized bonds in parallel. An alternative (and more precise, since commutation aspects are taken into account at the cell level) procedure is to renormalize the whole cell. This point is extensively discussed in Ref. ? , where it is shown that treating the cell "by pieces" gives good qualitative results for ferromagnetic quantum models, when compared to renormalizing the whole cell. The comparison between these two procedures has also been made for antiferromagnetic quantum models [? ] and again the agreement is quite good (note, however, that for models such that frustration effects are present, the two approaches give different qualitative results [? ])). In this work, we have applied the two procedures outlined above for $b=2$; the qualitative (and sometimes even the quantitative) agreement is excellent and put the Migdal-Kadanoff approximation made for $b=3$ on a firmer basis.

The hierarchical lettices we treat here are those with $b=2, d=2$ and 3, (see Fig. 1) and $b=3, d=2$ and 3 (see Fig. (2). The corresponding results are:

(a) $b=2, d=2$ : in this case, the results are the same treating the cell as a whole or within the MigdalKadanoff approximation. The critical temperature, $T_{c}$, and the correlation-length's critical exponent, $\nu$, are known exactly for the two-dimensional uniform model, namely $T_{c}=0$ [? ] and $\nu=\infty$. We obtain these exact results with our procedure. Therefore, according to the Harris-Luck criterion, Eq. (1), the crossover exponent is negative for any sequence. In fact, the eigenvalues for this model are: $\Lambda_{1}=1$ and $\Lambda_{2}=-1 / 2$. The former corresponds to the pure-model's critical behavior; the second determines the irrelevance of the aperiodicity, as predicted by the Harris-Luck criterion. The negative sign of the second largest eigenvalue is a signature of aperiodic systems [?];

(b) $b=2, d=3$ : in this case, we have $I$ ) renormalized the cell as a whole and $I I$ ) used the MigdalKadanoff approximation, as in the last item. In both procedures we obtain a finite critical temperature $\left(T_{c}=2.70\right.$ for the former and $T_{c}=2.91$ for the latter), as expected. The approximated value for the critical exponent $\nu$ is: $I$ ) 1.511, and $I I$ ) 1.398. Since $\omega=0$ for the period-doubling sequence, the crossover exponent is negative in both approximations, namely: $I) \phi=-0.511$, and $I I$ ) $\phi=-0.398$. Therefore, we expect the aperiodicity to be irrelevant. The eigenvalues of the matrices of the LRGE are: $I) \Lambda_{1}=1.582 ; \Lambda_{2}=-0.791$; and II) $\Lambda_{1}=1.642 ; \Lambda_{2}=-0.821$. As expected, in both cases the aperiodicity is irrelevant and the smaller eigenvalue is nagative, as commented above;

(c) $b=3, d=2$ : again, $T_{c}=0$ and $\nu=\infty$ for the uniform model. Our procedure (see Fig. 2) obtains the correct critical temperature but the value obtained for $\nu$ is an excellent approximation but not the exact one, namely $\nu=8.494$. As discussed above, we have to resort to the Migdal-Kadanoff procedure, in this case, but, in view of the comparison made in $(a)$ and $(b)$, we believe that the physical 
behavior is correctly described by this approximation. For this lattice, we have studied sequences $(i i)$ and (iii) (defined in Section III). For sequence (ii), the wandering exponent is $\omega=\ln (2) / \ln (3)$ and the crossover exponent is $\phi=-2.135$, i.e., the aperiodicity is irrelevant, according to the Harris-Luck criterion; the situation is analogous for sequence (iii), where $\omega=0$ and then $\phi=-7.494$ : the aperiodicity defined by this sequence is also irrelevant. For the eigenvalues of the substitutional matrices we obtain: $(i i): \Lambda_{1}=0.879 ; \Lambda_{2}=-0.654 ;(i i i)$ : $\Lambda_{1}=0.879 ; \Lambda_{2}=-0.224$. Therefore, the aperiodicity is irrelevant for both cases, as predicted by the Harris-Luck criterion. Note that, since the relevant fixed point is at $K_{A}^{*}=K_{B}^{*}=\infty$, the value $\Lambda_{1}=0.879$ means that this point unstable, in the renormalization-group sense;

(d) $b=3, d=3$ : we have used the Migdal-Kadanoff approximation and obtained $T_{c}=1.92$ and $\nu=1.551$ for the uniform case. Recalling the values for the wandering exponents for sequences (ii) and (iii) (see $(c)$ above), the crossover exponents are given by $\phi=0.427$ and $\phi=-0.551$, respectively. The eigenvalues of the matrix of the LRGE are: (ii): $\Lambda_{1}=2.030 ; \Lambda_{2}=-1.363 ;($ iii $): \Lambda_{1}=2.030$; $\Lambda_{2}=-0.667$. Therefore, sequence $(i i)$ is relevant and sequence (iii) is irrelevant, again in accordance with the Harris-Luck criterion. For the relevant case, a fixed cycle of period two emerges, as already found in Ref. ? . The location of this stable two-cycle is $\left(K_{A}^{*}=0.417, K_{B}^{*}=3.90\right)$; $\left(K_{A}^{*}=1.85, K_{B}^{*}=0.325\right)$. The eigenvalues of the LRGE associated with this double iteration are $\Lambda_{1}=3.89 ; \Lambda_{2}=0.250$. The specific heat critical exponent associated with the aperiodic fixed cycle, $\alpha_{a}$, is calculated from the relation:

$$
\alpha_{a}=2-d \frac{\ln b^{2}}{\ln \Lambda_{1}},
$$

where $d$ is the fractal dimension of the hierarchical lattice and $b$ is the scaling parameter associated with onde iteration. In this case, we obtain $\alpha_{a}=$ -2.855 , which is smaller than its counterpart for the uniform model $\alpha_{u}=-2.653$ (this value can be obtained from the value of $\nu$, quoted above, and the relation $\left.\alpha_{u}=2-d \nu\right)$.

We have also studied the classical isotropic Heisenberg model at low temperatures. Only in this limit this model is closed upon application of the renormalizationgroup transformation. Our procedure is appropriate to the study of two-dimensional systems, since only in these cases the non-trivial fixed point is at zero temperature. The renormalized parameters are [? ]:

$$
\frac{1}{K_{A}^{\prime}}=\frac{1}{b}\left(\frac{n_{1}}{K_{A}}+\frac{n_{2}}{K_{B}}\right) ; \frac{1}{K_{B}^{\prime}}=\frac{1}{b}\left(\frac{n_{3}}{K_{A}}+\frac{n_{4}}{K_{B}}\right) .
$$

These equations assume an aperiodic sequence built by the substitution rules:

$$
A \rightarrow \overbrace{A A \cdots A}^{n_{1}} \overbrace{B \cdots B}^{n_{2}} ; \quad B \rightarrow \overbrace{A A \cdots A}^{n_{3}} \overbrace{B \cdots B}^{n_{4}} .
$$

Note that, since the model is classical, the order of the interactions in Eqs. (11) is not relevant and the proportinality between the matrix of the LRGE and $\mathcal{M}^{T}$ holds in this case. It is easy to show that, for $K_{A}^{*}=K_{B}^{*}=\infty$, the matrix of the LRGE is given by:

$$
\left(\begin{array}{ll}
b n_{1} /\left(n_{1}+n_{2}\right)^{2} & b n_{2} /\left(n_{1}+n_{2}\right)^{2} \\
b n_{3} /\left(n_{3}+n_{4}\right)^{2} & b n_{4} /\left(n_{3}+n_{4}\right)^{2}
\end{array}\right) .
$$

Since $n_{1}+n_{2}=n_{3}+n_{4}=b$ and $n_{4}=0$ in the sequences studied here, the eigenvalues are $\lambda_{1}=n_{3} / b=1$ and $\lambda_{2}=-n_{2} / b$, with $\left|\lambda_{2}\right|<1$. So, the eigenvalues of the substutitional matrix are $\Lambda_{1}=b$ and $\Lambda_{2}=-n_{2}$, and the wandering exponnet is given by:

$$
\omega=\frac{\ln n_{2}}{\ln b}<1 .
$$

Note that the value of $\lambda_{1}$ implies that $\nu=\infty$ and, since $\omega<1$, any aperiodic sequence with $n_{1}+n_{2}=n_{3}+n_{4}=b$ will be irrelevant for the classical isotropic Heisenberg model in two dimensions. This result is supported by the formalims we apply in this work, since $\left|\lambda_{2}\right|<1$.

\section{SUMMARY}

Witihin a real-space renormalization-group framework, we have studied the quantum anisotropic Heisenbeg model with interactions following three different aperiodic sequences, on four different hierarchical lattices. We obtain the exact result $T_{c}=0$ for two-dimensional lattices, while our evaluation of $T_{c}$ is always finite when $d=3$. In accordance with symmetry arguments, the isotropic Heisenberg-model subsapce in an invariant one and the flow of the anisotropic models is always towards the Ising subspace. Our procedure allows for the calculation of the stability of the homogeneous fixed points, which agrees with the Harris-Luck criterion in all studied cases. For the relevant sequence, we established the presence of a new stable fixed cycle of period two and calculated its sepcific heat critical exponent. We also applied a low-temperature renormalization-group calculation to the isotropic classical Heisenberg model in twodimensional lattices: the results we obtain are exact on the respective hierarchical lattices and on this range of temperatures. We obtain that, on two-dimensional hierarchical lattices, all aperiodic sequences are irrelevant, in agreement with the Harris-Luck criterion.

\section{Acknowledgments}

The authors would like to thank FAPESC, FAPEAM, $\mathrm{CNPq}$, and CAPES for partial financial support. 